\title{
BMJ Open Determining cost-effectiveness of lung cancer screening in urban Chinese populations using a state-transition Markov model
}

\author{
Chengyao Sun, ${ }^{1}$ Xin Zhang, ${ }^{1}$ Sirou Guo, ${ }^{2}$ Yang Liu, ${ }^{3}$ Liangru Zhou, ${ }^{1}$ Jufang Shi, ${ }^{4}$
} Ning Wu, ${ }^{5}$ Zhao Zhai, ${ }^{6}$ Guoxiang Liu (D) ${ }^{1}$

To cite: Sun C, Zhang $X$, Guo $S$, et al. Determining cost-effectiveness of lung cancer screening in urban Chinese populations using a state-transition Markov model. BMJ Open 2021;11:e046742. doi:10.1136/ bmjopen-2020-046742

- Prepublication history for this paper is available online. To view these files, please visit the journal online (http://dx.doi org/10.1136/bmjopen-2020046742).

Received 10 November 2020 Accepted 14 June 2021

Check for updates

(C) Author(s) (or their employer(s)) 2021. Re-use permitted under CC BY-NC. No commercial re-use. See rights and permissions. Published by BMJ.

For numbered affiliations see end of article.

Correspondence to Professor Guoxiang Liu; Igx6301@163.com and Dr Xin Zhang;

zhangxinzhx0801@126.com

\section{ABSTRACT}

Objectives This study analyses the cost-effectiveness of annual low-dose CT (LDCT) screening of high-risk cancer populations in Chinese urban areas.

Design We used a Markov model to evaluate LDCT screening from a sociological perspective.

Setting The data from two large lung cancer screening programmes in China were used.

Participants The sample consisted of 100000 smokers who underwent annual LDCT screening until age 76. Intervention The study comprises five screening strategies, with the initial screening ages in both the screening strategies and their corresponding nonscreening strategies being $40,45,50,55$ and 60 years, respectively.

Primary and secondary outcome measures The incremental cost-effectiveness ratio (ICER) between screening and non-screening strategies at the same initial age was evaluated.

Results In the baseline scenario, compared with those who were not screened, the specific mortality from lung cancer decreased by $18.52 \%-23.13 \%$ among those who underwent screening. The ICER of LDCT screening ranges from US\$13056.82 to US\$15736.06 per quality-adjusted life year, which is greater than one but less than three times the gross domestic product per capita in China. An initial screening age of 55 years is the most cost-effective strategy.

Conclusions Baseline analysis shows that annual LDCT screening of heavy smokers in Chinese urban areas is likely to be cost-effective. The sensitivity analysis reveals that sensitivity, specificity and the overdiagnosis rate influence the cost-effectiveness of LDCT screening. All scenarios tested demonstrate cost-effectiveness, except for the combination of worst values of sensitivity, specificity and overdiagnosis. Therefore, the costeffectiveness of a screening strategy depends on the performance of LDCT screenings.

\section{INTRODUCTION}

According to GLOBOCAN statistics, there were 18.1 million new cancer cases and 9.6 million cancer-related deaths worldwide in 2018. Among them, lung cancer was the most commonly diagnosed cancer $(11.6 \%)$ and
Strengths and limitations of this study

In this study, a state-transition Markov model is developed to simulate the natural history of lung cancer development.

- Five screening strategies for lung cancer, and their corresponding non-screening strategies, are compared for cost and effectiveness, under a decision analytical model based on the Markov model.

- The model simulation includes cost-related parameters (screening, diagnosis and treatment), effectrelated parameters (quality-adjusted life years) and parameters related to transition probability (morbidity, mortality, clinical stage distribution of cancers, etc).

- The study assumes that all the people will voluntarily engage in the screening process.

- The cost-effectiveness of incidental findings is not included in this study.

the leading cause of cancer-related deaths $(18.4 \%) .^{1}$ In China alone, in 2014, there were 3.804 million new cancer cases and 2.296 million cancer-related deaths. Lung cancer was the most commonly diagnosed cancer $(20 \%)$ in China, and the leading cause of cancer-related death $(27.3 \%){ }^{2}$

In the past 30 years, the survival rate of individuals with lung cancer has only moderately improved. ${ }^{3}$ Even in developed countries, the 5-year overall survival rate for patients with lung cancer is approximately $15 \%-18 \%{ }^{4}$ However, the 5-year survival rate for patients with stage I lung cancer undergoing surgical resection is much higher at more than $70 \% .^{5}$ This highlights the importance of early detection and treatment of lung cancer. ${ }^{6}$

Common lung cancer screening methods include low-dose CT (LDCT) screening and chest X-rays. Currently, due to the low sensitivity and specificity, X-ray is not recommended by screening guidelines. ${ }^{7}$ LDCT screening has proven effective in reducing 
lung cancer mortality. ${ }^{9} 10$ The National Lung Screening Trial (NLST) in the USA showed that LDCT screening can reduce the specific mortality of lung cancer by $20 \%$ with only three annual screens. ${ }^{9}$ Subsequently, the lung cancer screening trial in Italy indicated that patients with stage I lung cancer tended to be more frequently diagnosed through annual LDCT screenings than the baseline. $^{10}$

Although the effectiveness of LDCT screening has been proven, economic evidence must be further explored. Scholars in many countries have conducted costeffectiveness analyses of lung cancer LDCT screening. The results suggest that it is cost-effective to carry out LDCT screening in high-risk lung cancer groups. ${ }^{11}$ However, most studies included in previous research papers came from developed countries. ${ }^{12}$ Moreover, at present, there are few studies on the cost-effectiveness of lung cancer screening in China. ${ }^{11}$

In this study, we combined data from two large lung cancer screening programmes in China (the National Lung Cancer Screening and Intervention Program (NLCSIP) and the Cancer Screening Program in Urban China (CanSPUC)), and lung cancer epidemiology data, ${ }^{14}$ to conduct a cost-effectiveness analysis of annual LDCT screening among high-risk populations in Chinese urban areas. We aimed to assess the cost-effectiveness of screening versus non-screening for lung cancer among these high-risk populations, and the ideal initial age to start screening from a cost-effectiveness perspective.

\section{MATERIALS AND METHODS}

\section{Study design}

In this study, a state-transition Markov model was developed to simulate the natural history of lung cancer development. In a Markov model, clinical states are described according to the discrete health states of individuals, the 'Markov states'. Individuals are always in one of these states, and all events of interest are modelled as a transition from one state to another. ${ }^{15}$ Based on the Markov model, we simulated screening and non-screening strategies to determine the costs and effectiveness of each strategy for individuals in a particular clinical state. The model simulation included cost-related parameters (screening, diagnosis and treatment), effect-related parameters (quality-adjusted life years; QALY) and parameters related to transition probability (morbidity, mortality, clinical stage distribution of cancers, etc). These parameters were derived from the NLCSIP and CanSPUC, published literature and cancer registry data. The study first conducts a baseline analysis and then a sensitivity analysis to assess the robustness of the results using reasonable ranges of uncertain parameters.

\section{Study population and screening strategies}

Five screening strategies and their corresponding nonscreening strategies for lung cancer are compared for cost and effectiveness under a decision analytical model

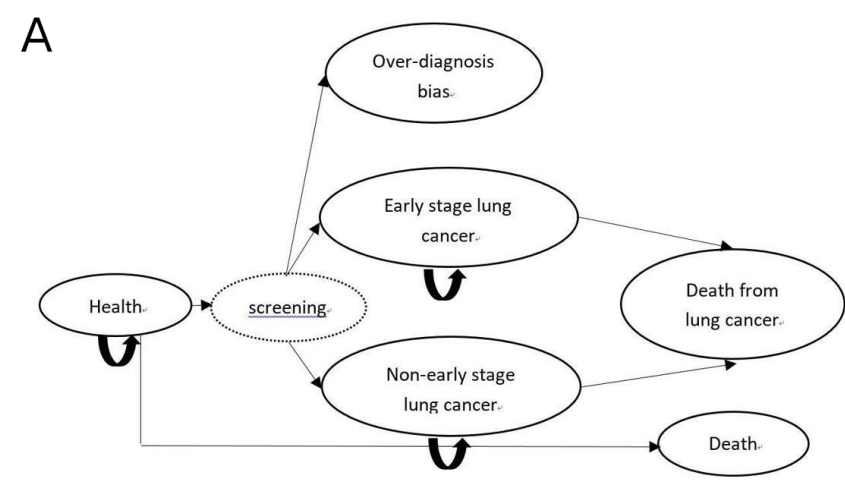

B

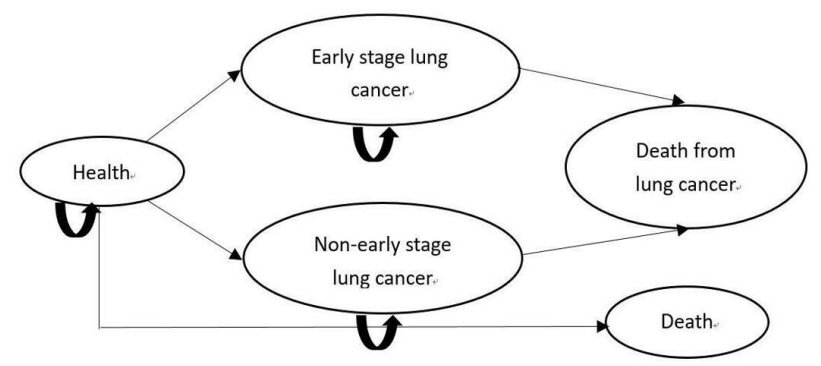

Figure 1 (A) Markov model of annual screening with lowdose CT (LDCT). (B) Markov model of non-screening group.

based on a state-transition Markov process. A hypothetical static cohort of 100000 high-risk smokers (>20 pack-years, where a pack-year refers to 20 cigarettes smoked every day for 1 year) from Chinese urban areas were entered into the model and their health histories were simulated by sex until 76 years of age. The last age of screening is 76 years old and follow-up is simulated until that age. The 100000 smokers are a 'closed block' cohort, which means no new entrants are allowed and exit is only through death. The proportion of males in this research was set according to the 2015 annual report of Chinese people and employment statistics. ${ }^{16}$ All health states are modelled as Markov states with a 1-year cycle. The initial screening ages for the five screening strategies and their corresponding nonscreening strategies are 40, 45, 50, 55 and 60 years. All individuals who enter the group are of the same age, and all of them go through LDCT screening. Several different scenarios were selected for the entry age, and 40,45 , etc represent different scenarios. We compared the costeffectiveness of screening and non-screening strategies at the same initial age.

\section{Markov model and its transition probabilities Non-screened cohort}

In the non-screening model, the natural history of lung cancer development is simulated as a transition from health to lung cancer (figure 1A). Using the data from the NLCSIP programme, we divided the cases of detection into early-stage (stage I) and non-early-stage lung cancer and, ultimately, death (death either from cancer or other causes).$^{17}$ The clinical stage distribution of patients with lung cancer in the non-screening cohort was determined based on a multicentre retrospective epidemiological 
survey from the CanSPUC programme. ${ }^{18}$ The probability of transition from health to death is derived from China's demographic census, ${ }^{19}$ while the probability of lung cancer-specific death is derived from the literature. ${ }^{20}$ The study determined the survival status of patients with lung cancer based on the registration and survival follow-up report data of 41802 lung cancer cases collected by the Shanghai Cancer Registry. The disease progression parameters from health to lung cancer are calculated based on the lung cancer incidence among smokers in China. ${ }^{14}$ The incidence among smokers (>20 pack-years) was calculated as follows.

First, age-specific and sex-specific rates of lung cancer incidence in the general Chinese urban population $\left(\mathrm{I}_{\mathrm{G}}\right)$ were collected from the China Cancer Registry Annual Report 2017 (urban data) at intervals of 5 years of age. ${ }^{14}$ In 2017, the National Central Cancer Registry (NCCR) collected data from local cancer registries regarding registrations in 2014. Based on the NCCR's data quality criteria, data submitted from 449 registries were checked and evaluated, with data from only 339 registries qualifying for the cancer registry annual report. All 339 cancer registries (140 in the eastern region, 112 in the central region and 87 in the western region) covered a total population of 288243347 (146 203891 males and 142 039456 females). Next, the lung cancer incidence rates among smokers $\left(I_{S}\right)$ in each age and gender group were calculated using formula $1^{21}$ :

$$
\mathrm{I}_{\mathrm{s}}=\mathrm{OR} \times \mathrm{I}_{\mathrm{G}} /(1+(\mathrm{OR}-1) \times \mathrm{R}),
$$

where OR (2.85 for men and 2.33 for women) is the odds ratio calculated from a meta-analysis of five casecontrol studies in China ${ }^{21}$ and $\mathrm{R}$ is the age-specific and sex-specific rate of smoking reported in the Global Adult Tobacco Survey China 2010 Country Report. ${ }^{22} \mathrm{I}_{\mathrm{G}}$ is the lung cancer incidence of the general population from the China Cancer Registry Annual Report 2017.

Further, we calculated the incidence of non-smokers $\left(\mathrm{I}_{\mathrm{N}}\right)$ based on the incidence of smokers $\left(\mathrm{I}_{\mathrm{S}}\right)$, the proportion of smokers and the overall population morbidity (I), according to formula 2 :

$$
\mathrm{I}=\mathrm{I}_{\mathrm{s}} \times \mathrm{R}+\mathrm{I}_{\mathrm{N}} \times(1-\mathrm{R})
$$

We used the relative risk (RR) of lung cancer among smokers ( $>20$ pack-years) versus non-smokers to calculate the lung cancer incidence $\left(I_{20}\right)$ of smokers $(>20$ packyears) in China using formula 3. The RR of lung cancer ( $>20$ pack-years) attributable to smoking is derived from published literature $^{23}$ (table 1):

$$
\mathrm{I}=\mathrm{I}_{\mathrm{s}} \times \mathrm{I}_{\mathrm{N}} \times(1-\mathrm{R}) .
$$

\section{Screened cohort}

Smokers in the screened cohort underwent annual LDCT testing and smokers with positive results were required to undergo additional diagnostic biopsy tests. The sensitivity and specificity of LDCT for lung cancer were set according to data from a published study. ${ }^{21}$ In addition, screening was superimposed on the lung cancer natural
Table 1 The lung cancer incidence grouped by age and gender among smokers

\begin{tabular}{lll}
\hline Incidence of smokers (age) & Male (\%) & Female (\%) \\
\hline $40-44$ & 0.03 & 0.05 \\
$45-49$ & 0.06 & 0.09 \\
$50-54$ & 0.13 & 0.14 \\
$55-59$ & 0.25 & 0.23 \\
$60-64$ & 0.40 & 0.35 \\
$65-69$ & 0.70 & 0.47 \\
$70-74$ & 0.93 & 0.66 \\
$75-76$ & 1.11 & 0.89 \\
\hline
\end{tabular}

history module, resulting in early detection as determined by LDCT screening performance characteristics (figure 1B).

The distribution of patients with lung cancer in the screened cohort was determined based on opportunistic multicentre screening data from the NLCSIP programme. The NLCSIP evaluated and screened 21397 high-risk lung cancer individuals from 18 provinces across the country. A total of 199 patients were diagnosed with lung cancer, of which $85.6 \%$ were stage I.

People who are screened may be diagnosed with lung cancer that does not cause clinical disease (overdiagnosis bias), while many of them may not be diagnosed under non-screening conditions. Previous studies set the overdiagnosis rate at $12 \%-20 \%$ based on early autopsy reports for lung cancer in Australia. ${ }^{24}$ The results of long-term follow-up of NLST considered that overdiagnosis could be avoided by histological type. ${ }^{25}$ Therefore, in base case analysis, the overdiagnosis rate is set as $0 \%$. We still consider the impact of overdiagnosis rate on the costeffectiveness in the sensitivity analysis.

In the model analysis, modelling lead time due to screening is important to avoid bias. Lead time, interpreted as the extended survival time due to screening, is the difference between the time of screening diagnosis and the time of clinical diagnosis. An average 1-year lead time for screening was incorporated into the study. ${ }^{18}$

\section{Model assumptions}

(1) People who participate in screening do not increase their cancer risk due to radiation from LDCT. According to the American Association of Physicists in Medicine, epidemiological evidence supporting increased cancer incidence or mortality from radiation doses below 100 $\mathrm{mSv}$ is inconclusive. ${ }^{26}$ (2) The compliance of the population for screening was $100 \%$ in the screening group, and participation will continue in annual lung cancer screening until the age of 76 years. (3) The clinical stage distribution of false-negative lung cancer diagnoses under the LDCT screening strategy is the same as that of nonscreened individuals. 


\section{Costs}

We consider the cost of screening, biopsy diagnosis and treatment. The cost of LDCT screening and lung cancer treatment is taken from the CanSPUC programme, and the cost of biopsy diagnosis is taken from the cost of medical services in hospitals with the CanSPUC programme. The treatment costs of patients with lung cancer are also collected from hospitals, multicentres and retrospective investigations. The questionnaire for the study includes three parts: basic information, clinical information and medical expenses information. Medical expenses are defined as the total hospitalisation expenses of patients with lung cancer, which is the medical expense information of a patient with lung cancer treated in the investigated hospital from admission to discharge. Data were collected from 40 hospitals in 13 provinces and 22 cities: Beijing, Guangdong (Dongguan, Foshan, Guangzhou, Shenzhen and Zhongshan), Shandong (Ji'nan), Jiangsu (Nantong and Xuzhou), Liaoning (Shenyang and Tieling), Zhejiang (Hangzhou and Ningbo), Hebei (Tangshan), Henan (Zhengzhou), Heilongjiang (Harbin and Daqing), Hunan (Changsha), Xinjiang (Urumqi), Gansu (Lanzhou and Jinchang), and included a total of 15437 persons whose treatment occurred-and who were discharged-between 1 January 2002 and 31 December 2011. All costs in this study are expressed in US dollars and are discounted to the price level in 2018 at a discount rate of $3 \%{ }^{27}$ (table 2$)$.

Table 2 Parameters used for the modelling of lung cancer screening protocols

\begin{tabular}{|c|c|c|}
\hline Variable & Values (range) & Reference \\
\hline $\begin{array}{l}\text { Proportion of early-stage cancer among lung cancers detected with } \\
\text { no screening }\end{array}$ & 19.00 & 17 \\
\hline $\begin{array}{l}\text { Proportion of early-stage cancer cases among lung cancers detected } \\
\text { with LDCT }\end{array}$ & 85.60 & NLCSIP \\
\hline Specificity of LDCT (\%) & $90.60(86.30-91.10)$ & 21 \\
\hline Mortality (\%) & & 20 \\
\hline Early-stage lung cancer & 11.12 & \\
\hline Non-early-stage lung cancer & 35.34 & \\
\hline $40-44$ & 59.30 & \\
\hline $45-64$ & 63.00 & \\
\hline $65-76$ & 40.20 & \\
\hline \multicolumn{3}{|l|}{ Women } \\
\hline $40-44$ & 1.60 & \\
\hline $45-64$ & 3.20 & \\
\hline $65-76$ & 6.70 & \\
\hline Cost (US\$) & & CanSPUC \\
\hline Utility & & 28 \\
\hline Early-stage lung cancer & 0.825 & \\
\hline Non-early-stage lung cancer & 0.573 & \\
\hline
\end{tabular}

CanSPUC, Cancer Screening Program in Urban China; LDCT, low-dose CT; NLCSIP, National Lung Cancer Screening and Intervention

Program; RR, relative risk. 
Quality of life year (QALY)

QALY takes into account both survival and the quality of life as determined by the progression and severity of lung cancer. We obtained utility scores of quality of life for each health state from a current meta-analysis. ${ }^{28}$ Utility scores were 0.823 for early-stage lung cancer, 0.573 for non-early-stage lung cancer and 1 for healthy status (table 2).

\section{Effectiveness of lung cancer screening}

The effectiveness of screening is measured by comparing the difference in lung cancer-specific deaths, life years and QALYs. The lung cancer-specific deaths and QALYs gained under each screening strategy are equal to the difference in their values between the screening strategy and its corresponding non-screening strategy.

The primary outcome of the cost-effectiveness analysis is the incremental cost-effectiveness ratio (ICER), which is calculated by dividing the incremental cost by the incremental QALYs gained under each screening strategy compared with its corresponding non-screening strategy. In this study, the ceiling ratio is defined as the threshold recommended by the WHO. ${ }^{29}$ When ICER is less than three times the gross domestic product (GDP) per capita, the strategy is cost-effective; otherwise not. China's percapita GDP in 2018 was US $\$ 9768.78$; thus, US $\$ 29306.34$ $(9768.78 \times 3)$ is the ceiling in our study.

\section{Sensitivity analysis}

The impact of parameters such as sensitivity, specificity and overdiagnosis rates was tested through a oneway sensitivity analysis to confirm the robustness of the results. The range of parameter variations was set as: $71.8 \%-100 \%$ for sensitivity, $86.3 \%-91.1 \%$ for specificity and $0 \%-20 \%$ for overdiagnosis rate. The worst scenario of LDCT screening is estimated by the combinations of the lowest values for sensitivity and specificity and the highest value for overdiagnosis rate.

\section{Patient and public involvement}

Patients or the public were not involved in the design, or conduct, or reporting or dissemination plans of our research.

\section{RESULTS}

Cost analysis

The screening strategy is obviously more expensive than the corresponding non-screening strategy owing to additional tests. The earlier the initial LDCT screening, the more it would cost regardless of the health outcomes. It was observed that screening for lung cancer is most expensive at the initial screening age of 40 years, costing US $\$ 194.30$ million. Screening at age 60 has the lowest cost at US\$113.88 million. The lower the initial screening age in the non-screening strategy, the less costly the screening is. The costs range from US $\$ 2.05$ million at the lower end to US $\$ 5.38$ million at the higher end for the five nonscreening strategies (table 3 ).

\section{Effectiveness}

The lower the initial screening age, the lower the number of lung cancer-specific deaths. When the initial screening age is 40 years, the number of lung cancer deaths is 6924 , a reduction of 1574 or $18.52 \%$ deaths compared with the corresponding non-screening strategy (8498 deaths from lung cancer). When the initial screening starts at 60 years, the number of lung cancer deaths is 5376, a reduction of $1617(23.13 \%)$ compared with the corresponding nonscreening strategy (6994 lung cancer deaths) (table 3). The earlier the initial screening age is, the more is the number of life years gained by the screening. The number of life years gained by the single years of age screening strategies is from 8532 to 13340 life years in comparison with its corresponding non-screening strategy. It was also observed that the earlier the initial screening among the five age groups, the more the QALYs gained. The

Table 3 Base case results comparing LDCT screening with no screening for different initial ages

\begin{tabular}{|c|c|c|c|c|c|c|c|}
\hline Initial age & Screen & $\begin{array}{l}\text { Cost } \\
\text { (million) }\end{array}$ & $\begin{array}{l}\text { Lung cancer } \\
\text { deaths }\end{array}$ & $\begin{array}{l}\text { Death } \\
\text { reduction (\%) }\end{array}$ & $\begin{array}{l}\text { Life year } \\
\text { gained }\end{array}$ & $\begin{array}{l}\text { QALY } \\
\text { (thousand) }\end{array}$ & $\begin{array}{l}\text { ICER } \\
\text { (US\$/QALY) }\end{array}$ \\
\hline 40 & Yes & 194.30 & 6924 & 18.52 & 16625 & 3350.51 & 15736.06 \\
\hline 45 & Yes & 176.96 & 6810 & 18.84 & 16226 & 2873.33 & 14647.50 \\
\hline 45 & No & 2.55 & 8391 & & & 2861.42 & \\
\hline 50 & No & 3.30 & 8228 & & & 2392.88 & \\
\hline 55 & Yes & 136.93 & 6167 & 20.72 & 13972 & 1950.27 & 13056.82 \\
\hline 55 & No & 4.27 & 7778 & & & 1940.11 & \\
\hline 60 & Yes & 113.88 & 5376 & 23.13 & 11297 & 1504.91 & 13473.15 \\
\hline 60 & No & 5.38 & 6994 & & & 1496.86 & \\
\hline
\end{tabular}

ICER, incremental cost-effectiveness ratio; LDCT, low-dose CT; QALY, quality-adjusted life year. 
Table 4 Effect of changing sensitivity and specificity of LDCT on ICER value of each screening strategy

\begin{tabular}{|c|c|c|c|c|c|c|}
\hline & & \multicolumn{5}{|c|}{ ICER (US\$/QALY) } \\
\hline & & $40^{*}$ & $45^{*}$ & $50 *$ & $55^{\star}$ & $60^{*}$ \\
\hline Sensitivity & $71.80 \%$ & 21783.45 & 20260.50 & 19015.37 & 18018.64 & 18714.41 \\
\hline \multirow[t]{2}{*}{ Specificity } & $86.30 \%$ & 17733.91 & 16507.20 & 15492.93 & 14714.83 & 15184.35 \\
\hline & $91.10 \%$ & 15503.75 & 14431.26 & 13544.44 & 12864.03 & 13274.17 \\
\hline
\end{tabular}

*Initial age of LDCT screening.

ICER, incremental cost-effectiveness ratio; LDC T, low-dose CT; QALY, quality-adjusted life year.

QALYs saved when screening occurred at ages 40, 45, 50, 55 and 60 , when compared with the corresponding nonscreening strategy, were 12 217; 11 907; 11 235; 10160 ; and 8053 , respectively (table 3 ).

\section{Incremental cost-effectiveness ratio}

As seen in table 3, the ICERs for lung cancer screening strategies at the ages of $40,45,50,55$ and 60 years are US $\$ 15$ 736.06; US $\$ 14$ 647.50; US $\$ 13$ 747.41; US\$13 056.82; and US $\$ 13473.15$, respectively. The most costeffective lung cancer screening strategy, corresponding to the smallest ICER, is to undertake screening at the age of 55 years. This indicates that per QALY saved in lung cancer screening should cost an additional US $\$ 13056.82$ at the initial age of 55 years.

\section{Sensitivity analysis}

Sensitivity

The sensitivity of LDCT screening is the percentage of people correctly diagnosed as patients with lung cancer based on the results. The ICER of screening strategies decreases as the sensitivity of the LDCT screening increases. The maximum and minimum ICERs of the five screening strategies are US $\$ 21783.45$ and US $\$ 10763.54$ per QALY, respectively, when sensitivity ranges from $71.8 \%$ to $100 \%$. According to the threshold recommended by the WHO, ${ }^{29}$ all screening strategies for lung cancer are cost-effective within the range of sensitivity of LDCT screening (table 4).

\section{Specificity}

The specificity in LDCT screening varies from $86.3 \%$ to $91.1 \%$. Similarly, the ICER of screening strategies decreases as the specificity increases in LDCT screening. All screening strategies for lung cancer are cost-effective within the range of specificity. The screening strategy starting at the age of 55 is the most cost-effective in terms of the ICERs (table 4).

\section{Overdiagnosis rate}

The diagnostic and treatment costs induced by overdiagnosis in lung screening will result in an increase in the incremental cost. An increase in the overdiagnosis rate is also associated with a decrease in incremental QALYs, since overdiagnosis worsens health outcomes. When the overdiagnosis rate is $0 \%$, the maximum and minimum
ICERs are US $\$ 13056.82$ and US $\$ 15736.06$ per QALY, respectively, in the five screening strategies. The ICER ranges from US $\$ 18499.35$ to US $\$ 23006.90$ per QALY in the five screening strategies when the overdiagnosis rate rises to $20 \%$ (figure 2 ).

\section{Multivariate sensitivity analysis}

In the worst-case scenario, the ICERs for lung cancer screening strategies at the ages of 40, 45, 50, 55 and 60 years are US\$43 458.23; US\$39 774.96; US\$36 895.34; US\$34 016.88; and US\$35 416.66, respectively (table 5). Accordingly, all screening strategies are not cost-effective based on the threshold of three times GDP per capita (US\$29 306.34). ${ }^{29}$ However, lung cancer deaths can be avoided in the worst-case scenario, compared with the base case scenario, if LDCT screening is adopted.

\section{DISCUSSION}

This study undertakes an economic evaluation of annual LDCT screening for lung cancer among high-risk smokers living in Chinese urban areas using cost-effectiveness and increment analysis. The results indicate that annual LDCT screening can reduce the number of deaths caused by lung cancer. This study shows that, compared with the corresponding non-screening strategy, the number of deaths caused by lung cancer was reduced by $18.52 \%$, $18.84 \%, 19.33 \%, 20.72 \%$ and $23.13 \%$ in the screening

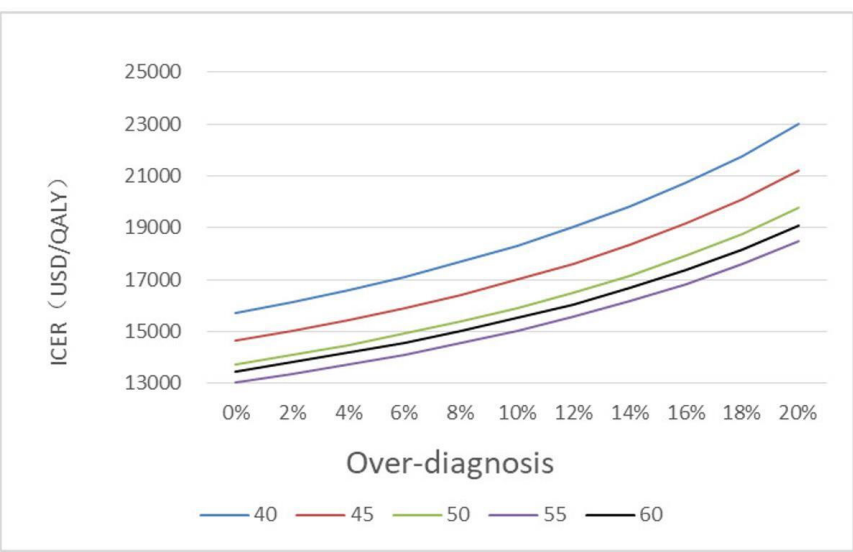

Figure 2 Effect of changing the overdiagnosis rate on the incremental cost-effectiveness ratio (ICER) values of each screening strategy. QALY, quality-adjusted life year. 
Table 5 Multivariate sensitivity analysis results comparing LDCT screening with no screening for different initial ages

\begin{tabular}{lllllll}
\hline Initial age & Screen & $\begin{array}{l}\text { Cost } \\
\text { (million) }\end{array}$ & $\begin{array}{l}\text { Lung cancer } \\
\text { deaths }\end{array}$ & $\begin{array}{l}\text { Death } \\
\text { reduction (\%) }\end{array}$ & $\begin{array}{l}\text { QALY } \\
\text { (thousand) }\end{array}$ & $\begin{array}{l}\text { ICER } \\
\text { (US\$/QALY) }\end{array}$ \\
\hline 40 & Yes & 217.90 & 7130 & 16.10 & 3343.26 & 43458.23 \\
\hline 40 & No & 2.05 & 8498 & & 3338.29 & 39774.96 \\
\hline 45 & Yes & 198.30 & 7020 & 16.34 & 2866.34 & 2861.42 \\
\hline 45 & No & 2.55 & 8391 & & 2397.58 & 36895.34 \\
\hline 50 & Yes & 176.65 & 6851 & 16.73 & 2392.88 & 1944.49 \\
\hline 50 & No & 3.30 & 8228 & & 17.83 & 1940.11 \\
\hline 55 & Yes & 153.33 & 6392 & & 1500.31 \\
\hline 60 & No & 4.27 & 7778 & 19.75 & 1496.86 \\
\hline
\end{tabular}

ICER, incremental cost-effectiveness ratio; LDCT, low-dose CT; QALY, quality-adjusted life year.

groups consisting of individuals aged 40, 45, 50, 55 and 60 years, respectively. The results of sensitivity analysis also illustrate that LDCT screening for lung cancer can reduce deaths by $16.10 \%-19.75 \%$ even under the most unfavourable circumstances. The NLST in the USA showed that LDCT screening can reduce the specific mortality of lung cancer by $20 \% .^{9}$ This is similar to the result of our study. However, the NLST conducted only three annual screens, whereas our research conducted decades of annual screens. The patients with cancer in this study had a longer follow-up period, which led to more deaths of patients with early lung cancer. Therefore, in the longterm follow-up, the effect of LDCT screening is more reflected in the increase in life years and QALYs.

Since younger smokers are less likely to develop lung cancer, using the screening in older initial age groups can reduce more deaths. However, in this study, QALY value and life years from the screening strategies decrease when the initial screening age increases. The QALYs at age 40 years were the most optimal, followed by the screening strategies at ages 45, 50, 55 and 60 years (table 3). However, the costs of screening decrease with an increase in the initial screening age.

All the costs in the five screening strategies are greater than those in the corresponding non-screening strategies. Healthy smokers in the screening group were requested to undergo annual LDCT screening for lung cancer. Furthermore, in some false-positive instances, healthy smokers-diagnosed by LDCT screening-had to undergo a biopsy, both of which contribute to the excessive costs of screening strategies.

Although the costs of screening groups are much higher than those of the corresponding non-screening groups, the health effect in the screening groups is greater than in the corresponding non-screening groups. Therefore, a prioritised screening strategy should be determined based on which strategy involves the lowest cost of screening while delivering the best health outcome.
It is necessary, then, to develop screening strategies for economic evaluation.

When considering both cost and effectiveness, the screening strategy with an initial age of 55 years showed the best cost-effectiveness. Screening cost-effectiveness progressively worsened in the following age order: 60, 50, 45 and 40. Even sensitivity analysis revealed that age 55 presented the least cost in acquiring a QALY, when compared with the non-screening groups (table 3).

Further, this study shows that the incremental cost and incremental effectiveness between the screening and non-screening strategies decrease as the initial age of the screening group increases, resulting in the ICER of each screening strategy first decreasing and then increasing as the initial age of the screening groups increases. Hence, the trend of incremental cost reduction is greater than that of incremental effectiveness reduction when the initial age of the screening group is no more than 55 years, and the reverse is true when the initial age is over 55 years. Therefore, the screening strategy with an initial age of 55 years is the most cost-effective for Chinese annual LDCT screening for lung cancer. The optimal screening strategy for lung cancer presented in our study is consistent with the screening guidelines provided by the US Preventive Services Working Group and Ontario Cancer Care Canada. ${ }^{30} 31$

When compared with other studies, the ICER value of screening strategies in this study is relatively lower. For instance, an American study based on NLST indicated that screening with LDCT costs an additional US\$81000 per QALY gained compared with no screening. ${ }^{32}$ Another study in Canada showed that the ICER value of annual LDCT screening compared with no screening was US\$52000 per QALY. ${ }^{33}$ The ICER value of screening strategies in these studies is far greater than ours (US\$13056US\$15 736/QALY). A previous study has proven that the ICER value was most sensitive to variations in LDCT examination costs. ${ }^{34}$ Compared with American research, 
we found that the costs of LDCT screening in our study are low (US\$285 for LDCT screening in American study and US\$68 in this one).

It can be concluded from the results of the single factor sensitivity analysis that the baseline results are reliable. Although the sensitivity and specificity of LDCT screening and overdiagnosis have a certain impact on the research results, the results regarding the cost-effectiveness of screening hold. When assuming the worst-case estimation of sensitivity, specificity and overdiagnosis of LDCT screening in the multiple sensitivity analysis, the ICER of screening strategies (US\$34016-US\$43 458/QALY) is greater than the tripled value of per-capita GDP (US $\$ 29$ 306/QALY); thus, there is no cost-effectiveness in such circumstances. However, convincing evidence for sensitivity, specificity and overdiagnosis has not been provided in China.

Most existing studies explore screening itself; however, effective screening strategies can yield far greater benefits than indicated in our estimations. For instance, first, the health education provided by the screening programme may encourage many smokers to quit smoking, thereby reducing the risk of lung cancer and other related diseases. Second, the patients saved by screening can continue to work and create value, which contributes to productivity, tax revenues and so on. Third, in addition to the contribution to patients' quality of life, there is also a positive impact on the quality of life of their relatives. Fourth, the current literature identifies other important benefits-diagnosis of kidney stones, uncertain renal masses, adrenal masses and abdominal aortic aneurysm. ${ }^{35}$ The cost-effectiveness of screening can be further improved by considering these factors.

There are some limitations to this study and some assumptions that can be modified in further studies. For example, the study assumes that all people will voluntarily engage in the screening process, and that some people may quit during the process, affecting the cost-effectiveness of screening. Consequently, further studies should address the issue of compliance. Second, we divided the distribution of cancer stages into early and non-early. This was mainly because of lack of high-quality studies from which we could draw robust estimates of relevant parameters. There was also a shift from late stage to mid-stage, which might underestimate the cost-effectiveness. Finally, the study assumes that the clinical stage distribution of falsenegative lung cancer in LDCT screening strategy is the same as that of unscreened individuals. The challenge of diagnosing a $5 \mathrm{~mm}$ nodule with imaging followed by biopsy is much harder than for larger, late-stage cancer. This is a conservative assumption.

\section{CONCLUSION}

In summary, we can conclude that annual LDCT screenings among high-risk smokers ( $>20$ pack-years) in urban areas of China are likely to be cost-effective. However, the effectiveness depends on the performance of the screening strategy. Among the five screening strategies, screening from the initial age of 55 years is found to be the most cost-effective.

\section{Author affiliations}

${ }^{1}$ Department of Health Economics, College of Health Management, Harbin Medical University, Harbin, China

${ }^{2}$ Medical insurance office, Tianjin Medical University Cancer Institute and Hospital, Tianjin, China

${ }^{3}$ Department of Health Education, College of Public Health, Harbin Medical University, Harbin, China

${ }^{4}$ Office of Cancer Screening, National Cancer Center/National Clinical Research Center for Cancer/Cancer Hospital, Chinese Academy of Medical Sciences and Peking Union Medical College, Beijing, China

${ }^{5}$ Department of Diagnostic Radiology, Cancer Hospital, Chinese Academy of Medical Sciences and Peking Union Medical College, Beijing, China

${ }^{6}$ Department of Gastrointestinal Surgery, Harbin Medical University Third Clinical College, Harbin, China

Acknowledgements The authors would like to express their gratitude to $\mathrm{XZ}$ for his valuable input at the earlier stages of model development.

Contributors Conception and design; acquisition of data; analysis and interpretation of data; drafting of the manuscript; administrative, technical, material support: CS, XZ and GL. Analysis and interpretation of data, statistical analysis: SG, YL and LZ. Critical revision of the manuscript for important intellectual content: JS, NW and ZZ. All authors have read and approved the final manuscript.

Funding This work was supported by the National Key R\&D Program of China (2017YFC1308700; 2017YFC1308705) and the National Natural Science Fund (general grant: 71673071).

Competing interests None declared.

Patient and public involvement Patients and/or the public were not involved in the design, or conduct, or reporting, or dissemination plans of this research.

Patient consent for publication Not required.

Ethics approval This study was approved by the Ethics Committee of the Cancer Institute and Hospital, Chinese Academy of Medical Sciences (15-070/997).

Provenance and peer review Not commissioned; externally peer reviewed.

Data availability statement Data are available in a public, open-access repository. Data are available upon reasonable request. Data sharing not applicable as all data sets analysed for this study (the data from the National Lung Cancer Screening and Intervention Program (NLCSIP) and the Cancer Screening Program in Urban China (CanSPUC)) are not published.

Open access This is an open access article distributed in accordance with the Creative Commons Attribution Non Commercial (CC BY-NC 4.0) license, which permits others to distribute, remix, adapt, build upon this work non-commercially, and license their derivative works on different terms, provided the original work is properly cited, appropriate credit is given, any changes made indicated, and the use is non-commercial. See: http://creativecommons.org/licenses/by-nc/4.0/.

ORCID iD

Guoxiang Liu http://orcid.org/0000-0002-4839-4307

\section{REFERENCES}

1 Bray F, Ferlay J, Soerjomataram I, et al. Global cancer statistics 2018: GLOBOCAN estimates of incidence and mortality worldwide for 36 cancers in 185 countries. CA Cancer J Clin 2018;68:394-424.

2 Chen W, Sun K, Zheng R, et al. Cancer incidence and mortality in China, 2014. Chin J Cancer Res 2018;30:1-12.

3 Siegel R, Naishadham D, Jemal A. Cancer statistics, 2012. CA Cancer J Clin 2012;62:10-29.

4 Tarver T. Cancer Facts \& Figures 2014. J Consum Health Internet 2012;16:366-7.

5 Liang W, Shao W, Jiang G, et al. Chinese multi-institutional registry (CMIR) for resected non-small cell lung cancer: survival analysis of 5,853 cases. J Thorac Dis 2013;5:726-9.

6 Wood DE, Eapen GA, Ettinger DS, et al. Lung cancer screening. J Natl Compr Canc Netw 2012;10:240-65. 
7 Wood DE, Kazerooni EA, Baum SL, et al. Lung cancer screening, version 3.2018, NCCN clinical practice guidelines in oncology. J Natl Compr Canc Netw 2018;16:412-41.

8 Zhou Q-H, Fan Y-G, Bu H, et al. China National lung cancer screening guideline with low-dose computed tomography (2015 version). Thorac Cancer 2015;6:812-8.

9 , Aberle DR, Adams AM, et al, National Lung Screening Trial Research Team. Reduced lung-cancer mortality with low-dose computed tomographic screening. N Engl J Med 2011;365:395-409.

10 Lopes Pegna A, Picozzi G, Falaschi F, et al. Four-Year results of lowdose CT screening and nodule management in the ITALUNG trial. $J$ Thorac Oncol 2013;8:866-75.

11 Liu CC, Shi JF, Liu GX, et al. [Cost-effectiveness of lung cancer screening worldwide: a systematic review]. Zhonghua Liu Xing Bing Xue Za Zhi 2019;40:218-26.

12 Field JK, Duffy SW, Baldwin DR, et al. Uk lung cancer RCT pilot screening trial: baseline findings from the screening arm provide evidence for the potential implementation of lung cancer screening. Thorax 2016;71:161-70.

13 Kumar V, Cohen JT, van Klaveren D, et al. Risk-Targeted lung cancer screening: a cost-effectiveness analysis. Ann Intern Med 2018;168:161-9.

14 HeJ, Chen W. China cancer registry annual report (2017) People's Medical Publishing House; 2018.

15 Sonnenberg FA, Beck JR. Markov models in medical decision making: a practical guide. Med Decis Making 1993;13:322-38.

16 . China population and employment statistics YEARBOOK. Beijing,China Department of Population and Employment Statistics of the National Bureau of Statistics of China; 2015.

17 Shi J-F, Wang L, Wu N, et al. Clinical characteristics and medical service utilization of lung cancer in China, 2005-2014: overall design and results from a multicenter retrospective epidemiologic survey. Lung Cancer 2019;128:91-100.

18 Mahadevia PJ, Fleisher LA, Frick KD, et al. Lung cancer screening with helical computed tomography in older adult smokers: a decision and cost-effectiveness analysis. JAMA 2003;289:313-22.

19 . Tabulation on the 2010 Population Census of the People's Republic of China. Beijing,China Department of Population and Employment Statistics of the National Bureau of Statistics of China; 2010.

20 Zhang Met al. Survival analysis of patients with lung cancer in Shanghai. China Oncology 2017.

21 Wang Z, Han W, Zhang W, et al. Mortality outcomes of low-dose computed tomography screening for lung cancer in urban China: a decision analysis and implications for practice. Chin $J$ Cancer 2017;36:57.

22 CDC. Global adult tobacco survey (GATS) China 2010 country report. Shanghai,China CDC China; 2011.

23 Osaki Y, Okamoto M, Kaetsu A, et al. Retrospective cohort study of smoking and lung cancer incidence in rural prefecture, Japan. Environ Health Prev Med 2007;12:178-82.

24 Manser R, Dalton A, Carter R, et al. Cost-Effectiveness analysis of screening for lung cancer with low dose spiral CT (computed tomography) in the Australian setting. Lung Cancer 2005;48:171-85.

25 National Lung Screening Trial Research Team. Lung cancer incidence and mortality with extended follow-up in the National lung screening trial. J Thorac Oncol 2019;14:1732-42.

26 American association of physicists in medicine. Available: https:// www.aapm.org/org/policies/details.asp?id=318\&type=PP\&current= true

27 Drummond MF, Jefferson TO. Guidelines for authors and peer reviewers of economic submissions to the BMJ. BMJ 1996;313:275-83.

28 Sturza J. A review and meta-analysis of utility values for lung cancer. Med Decis Making 2010;30:685-93.

29 World Bank. Official exchange rate, 2018. Available: http://www. worldbank.org

30 Roberts H, Walker-Dilks C, Sivjee K, et al. Screening high-risk populations for lung cancer: guideline recommendations. $J$ Thorac Oncol 2013;8:1232-7.

31 Moyer VA, U.S. Preventive Services Task Force. Screening for lung cancer: U.S. preventive services Task force recommendation statement. Ann Intern Med 2014;160:330-8.

32 Black WC, Gareen IF, Soneji SS, et al. Cost-Effectiveness of CT screening in the National lung screening trial. N Engl J Med 2014;371:1793-802.

33 Goffin JR, Flanagan WM, Miller AB, et al. Biennial lung cancer screening in Canada with smoking cessation - outcomes and costeffectiveness. Lung Cancer 2016;101:98-103.

34 ten Haaf K, Tammemägi MC, Bondy SJ, et al. Performance and cost-effectiveness of computed tomography lung cancer screening scenarios in a population-based setting: a Microsimulation modeling analysis in Ontario, Canada. PLoS Med 2017;14:e1002225.

35 Swensen SJ, Jett JR, Sloan JA, et al. Screening for lung cancer with low-dose spiral computed tomography. Am J Respir Crit Care Med 2002;165:508-13. 